\title{
Adaptive BLAST Decision-Feedback Equalizer FOR MIMO-FBMC/OQAM Systems
}

\author{
Eleftherios Kofidis \\ Department of Statistics and Insurance Science \\ University of Piraeus \\ 18534 Piraeus, Greece. \\ Email: kofidis@unipi.gr
}

\author{
Athanasios A. Rontogiannis \\ Institute for Space Applications and Remote Sensing \\ National Observatory of Athens \\ 15236 P. Penteli, Greece. \\ Email: tronto@space.noa.gr
}

\begin{abstract}
Filter bank-based multicarrier modulation (FBMC) using offset quadrature amplitude modulation (OQAM), known as FBMC/OQAM, provides an attractive alternative to the conventional cyclic prefix-based orthogonal frequency division multiplexing (CP-OFDM), especially in terms of increased robustness to frequency offset and Doppler spread, and high bandwidth efficiency. However, channel equalization in FBMC/OQAM is a nontrivial task, mainly because of the fact that the subchannels are no longer flat, in general. In multiple-antenna (MIMO) time-varying systems, equalizing the channel becomes even more challenging. This paper presents an adaptive $T / 2$-spaced decision-feedback equalization (DFE) algorithm for MIMO-FBMC/OQAM systems, that is both computationally efficient and numerically stable. Its structure follows the V-BLAST idea and the algorithm is applied in a per subcarrier fashion. Simulation results are reported that demonstrate its effectiveness in time-varying MIMO channels with high frequency selectivity.
\end{abstract}

\section{INTRODUCTION}

Orthogonal frequency division multiplexing (OFDM) is currently enjoying popularity in both wired and wireless communication systems, mainly because of its immunity to multipath fading, which allows for a significant increase in the transmission rate [21]. With the aid of a cyclic prefix (CP), OFDM can "reform" a frequency selective channel into a set of parallel flat channels with independent noise disturbances. This greatly simplifies both the channel estimation and equalization tasks. However, these advantages come at the cost of an increased sensitivity to frequency offset and Doppler spread. This is due to the fact that, although the subcarrier functions are perfectly localized in time, they suffer from spectral leakage in the frequency domain. Moreover, the inclusion of the $\mathrm{CP}$ entails a loss in spectral efficiency, which, in practical systems, can become as high as $25 \%$ [2].

Filter bank-based multicarrier modulation (FBMC) using offset quadrature amplitude modulation (OQAM), known as FBMC/OQAM or OFDM/OQAM [13], provides an alternative to $\mathrm{CP}-\mathrm{OFDM}$, that can mitigate these drawbacks. FBMC/OQAM employs pulse shaping via an IFFT/FFT-based efficient filter bank, and staggered OQAM symbols, i.e., real symbols at twice the symbol rate of OFDM/QAM, are loaded on the subcarriers [18]. This allows for the pulses to be well

This work was supported in part by an FP7 grant, project PHYDYAS (http://www.ict-phydyas.org). localized in both the time and the frequency domains. As a consequence, the system's robustness to frequency offsets and Doppler effects is increased and at the same time an enhanced spectral containment, for bandwidth sensitive applications (e.g., cognitive radio [1]), is offered. Moreover, FBMC/OQAM does not require the inclusion of a $\mathrm{CP}$, which may lead to even higher transmission rates [18]. ${ }^{1}$

However, the previously mentioned advantages of FBMC/OQAM come at the cost of subcarrier functions being now orthogonal only in the real field, which means that there is always an intrinsic imaginary interference among (adjacent) subcarriers [11]. Moreover, the subchannels cannot, in general, be modeled as frequency flat, especially in timevarying environments where the number of subcarriers has to be small enough [8]. This makes signal processing tasks for FBMC/OQAM systems, such as channel equalization, more challenging, compared to OFDM/QAM.

Using multiple antennas at the transmitter and/or receiver of an FBMC/OQAM system can result in further significant increases in bandwidth efficiency, in a manner analogous to MIMO-OFDM systems [19]. The presence of inter-antenna interference, though, makes signal processing in a MIMOFBMC/OQAM system even more challenging. The literature on equalization for multi-antenna FBMC/OQAM systems is quite limited and mostly restricted to mildly frequency selective channels with frequency flat subchannels. The more interesting case of frequency selective subchannels has only recently been addressed [10]. In that work, the per-subcarrier minimum mean squared error (MMSE) linear equalizer is first derived, in the time domain, and then extended to a so-called ordered successive interference cancellation (OSIC) scheme, which follows the V-BLAST idea [7]. The timevarying scenario was not addressed in [10] and, to the best of our knowledge, adaptive solutions to the problem of FBMC/OQAM equalization have so far appeared only for single-antenna systems (see, e.g., [22] and the references therein).

In this paper, an adaptive decision-feedback equalization (DFE) algorithm for MIMO-FBMC/OQAM systems is pre-

\footnotetext{
${ }^{1}$ Nevertheless, this advantage was partly given up in [14] and a CP-based OFDM/OQAM system was proposed for the sake of facilitating the data reception process.
} 


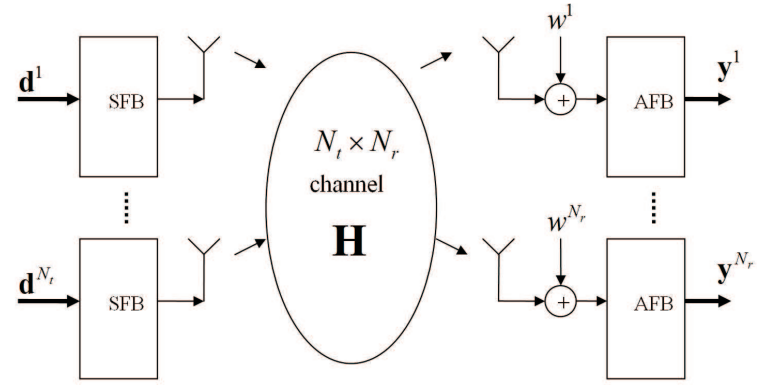

Fig. 1. The MIMO-FBMC/OQAM system.

sented and its performance is evaluated through simulation results. The equalizer's structure is dictated by the BLAST idea and its equivalence with the generalized DFE [9]. Such a DFE, based on efficient RLS, was first developed in [5], for flat fading channels, and later extended to frequency selective channels in [12]. In the latter work, an alternative parameterization, based on the Cholesky factorization of the data autocorrelation matrix, was also derived and shown to result in a more computationally efficient and numerically stable version. The $T / 2$-spaced DFE presented in this paper is an adaptation of the scheme of [12] to FBMC/OQAM, applied in a per subcarrier fashion. Simulation results are presented that demonstrate the effectiveness of the algorithm, in timevarying environments and, particularly, in highly frequency selective channels.

The rest of the paper is organized as follows. The MIMOFBMC/OQAM system is described in Section II. Section III is devoted to the presentation of the adaptive DFE algorithm, with emphasis on the Cholesky-based parameterization. Simulation results are reported and discussed in Section IV. Section V concludes the paper.

\section{THE MIMO-FBMC/OQAM SySTEM}

Consider a MIMO-FBMC/OQAM system with $N_{t}$ transmit and $N_{r}$ receive antennas, as in Fig. 1. A spatial multiplexing (SM) configuration is assumed, that is, the transmit antennas are fed with independent streams. Each transmit stream is the result of a synthesis filter bank (SFB), while each received signal is demodulated with an analysis filter bank (AFB). ${ }^{2}$ The SFB inputs consist of $T / 2$-spaced real symbols, corresponding to staggered real and imaginary parts of complex QAM symbols. The transmitted signals undergo multipath fading, modeled as a (highly) frequency selective, time-varying channel. At the receiver front end, noise $(w)$ is added, which is assumed to be white, both temporally and spatially.

Let $M$ be the number of subcarriers in each of the FBMC/OQAM modulators/demodulators. Denote by

$$
\boldsymbol{y}_{k, n}=\left[\begin{array}{llll}
y_{k, n}^{1} & y_{k, n}^{2} & \cdots & y_{k, n}^{N_{r}}
\end{array}\right]^{T}
$$

\footnotetext{
${ }^{2}$ With exponentially modulated filters, the filter banks can be efficiently realized via IFFT (SFB) and FFT (AFB), as shown in [18].
}

the outputs of the $N_{r}$ AFBs at frequency $k=0,1, \ldots, M-1$ and time $n$ and let

$$
\boldsymbol{d}_{k, n}=\left[\begin{array}{llll}
d_{k, n}^{1} & d_{k, n}^{2} & \cdots & d_{k, n}^{N_{t}}
\end{array}\right]^{T}
$$

be the corresponding real inputs to the $N_{t}$ SFBs. The noise components in $y_{k, n}^{q}$, s, $q=1,2, \ldots, N_{r}$, are clearly uncorrelated to each other. However, each of them is (stationary and) colored [4]. It can, nevertheless, be seen that, in practice and for well-designed filter banks, these noise terms are only weakly colored. Hence, for the sake of simplicity and to avoid overly increasing the frequency selectivity of the subchannels through the use of whitening filters, we will henceforth assume that the noise at the AFB outputs is white. ${ }^{3}$

Due to the good frequency localization of the filters used, $\boldsymbol{y}_{k, n}$ will only depend on the inputs to the $k$ th subcarrier and its adjacent subcarriers, $k \pm 1$, while the interference from further subcarriers can be neglected [10]. ${ }^{4}$ In this work, we will make the further assumption that we can also neglect the interference from the adjacent subcarriers, an assumption also made in earlier related works [10], [22]. The reason behind this is that viewing the equalization problem on a per subcarrier basis greatly simplifies BLAST detection since the optimal ordering can vary from one subcarrier to another.

Nevertheless, the $M$ subchannels are, in general, frequency selective, unlike what happens in the MIMO-OFDM system with long enough CP [19]. The assumption of flat subchannels, made e.g. in [6], can only hold true when the channel is mildly frequency selective and/or the filters employed are sufficiently well localized. The latter translates to a large number of subcarriers and implies a high computational cost for the FBMC/OQAM transmultiplexer. Moreover, keeping the effects from the frequency dispersion low in time-varying enviroments requires the number of subcarriers to be sufficiently small, hence resulting in frequency selective subchannels. In this paper, we will address this more realistic scenario, involving a wideband MIMO fast fading channel with subchannels that are frequency selective.

\section{AdAptive RLS-BASEd DeCision-FeEdBACK EQUALIZER}

In this section, we present an adaptive decision-feedback equalization (DFE) scheme for the MIMO-FBMC/OQAM system, implementing the V-BLAST idea. This is based on the well-known equivalence of V-BLAST with the generalized DFE (GDFE) [9] and involves a MIMO DFE per subcarrier. The block structure of the DFE for the $k$ th subcarrier is shown in Fig. 2. For each subcarrier, the equalizer consists of $N_{t}$ serially connected stages, each one equalizing one of the $N_{t}$ symbol streams according to BLAST ordering, which is adaptively updated. Both the DFE coefficients and the stream ordering are updated via an RLS-based algorithm.

\footnotetext{
${ }^{3}$ An assumption also made, tacitly, in [22].

${ }^{4}$ In theory, a subcarrier is interfered by all the others, and hence, even for a single-antenna FBMC/OQAM system, the equalizer should, in principle, be designed as MIMO [15].
} 


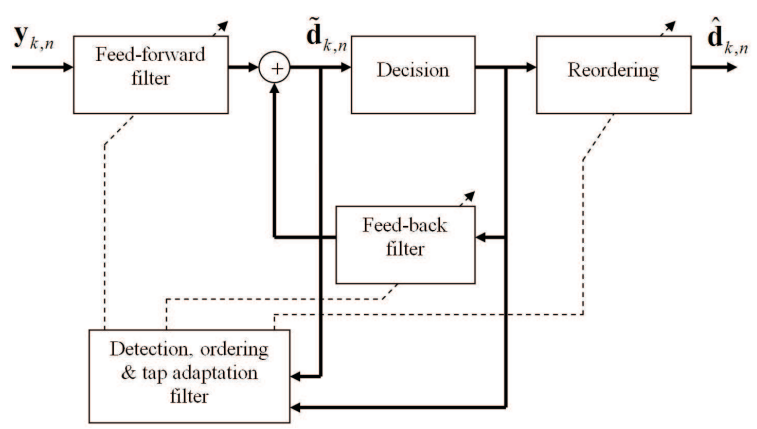

Fig. 2. Structure of the MIMO-FBMC/OQAM adaptive DFE for the $k$ th subcarrier.

Such a MIMO DFE was originally presented in [5], for (single carrier) flat fading channels. It was later demonstrated [17] that the algorithm in [5] may diverge after a number of iterations due to numerical instability. A numerically stable version was developed in [17], based on the square-root factorization of the DFE input autocorrelation matrix. In order to apply this algorithm in a MIMO-FBMC system, its extension to frequency selective channels is required. Such a frequency selective version of the algorithm of [17] was presented in [12] and it retains the nice numerical behavior of its flat counterpart. This scheme is the basis of the MIMOFBMC/OQAM DFE presented below, properly adapted to the FBMC/OQAM context.

Consider a subcarrier $k$. Each of the $N_{t}$ stages of the associated equalizer, say stage $p$, is a DFE consisting of a feed-forward filter of length $L_{f} N_{r}, \boldsymbol{f}_{k, n}(p)$, and a feed-back filter of length $L_{b} N_{t}+p-1, \boldsymbol{b}_{k, n}(p)$. The DFE output is given by:

$$
\begin{aligned}
\tilde{d}_{k, n}^{o_{p}} & =\Re\{\underbrace{\left[\boldsymbol{f}_{k, n}^{H}(p) \boldsymbol{b}_{k, n}^{H}(p)\right]}_{\boldsymbol{w}_{k, n}^{H}(p)} \boldsymbol{y}_{k, n}(p)\} \\
& =\Re\left[\boldsymbol{w}_{k, n}^{H}(p) \boldsymbol{y}_{k, n}(p)\right]
\end{aligned}
$$

where $o_{p}$ denotes the index of the stream detected at this stage, and

$$
\boldsymbol{y}_{k, n}(p)=\left[\begin{array}{ll}
\overline{\boldsymbol{y}}_{k, n}^{T} & \hat{\boldsymbol{d}}_{k, n}^{T}(p)
\end{array}\right]^{T}
$$

with

$$
\overline{\boldsymbol{y}}_{k, n}=\left[\begin{array}{llll}
\boldsymbol{y}_{k, n-L_{f}+1}^{T} & \boldsymbol{y}_{k, n-L_{f}}^{T} & \cdots & \boldsymbol{y}_{k, n}^{T}
\end{array}\right]^{T},
$$

and

$\hat{\boldsymbol{d}}_{k, n}(p)=\left[\begin{array}{llllll}\hat{\boldsymbol{d}}_{k, n-L_{b}}^{T} & \cdots & \hat{\boldsymbol{d}}_{k, n-1}^{T} & \hat{d}_{k, n}^{o_{1}} & \cdots & \hat{d}_{k, n}^{o_{p-1}}\end{array}\right]^{T}$,

where

$$
\hat{\boldsymbol{d}}_{k, l}=\left[\begin{array}{llll}
\hat{d}_{k, l}^{1} & \hat{d}_{k, l}^{2} & \cdots & \hat{d}_{k, l}^{N_{t}}
\end{array}\right]^{T}
$$

and $\hat{d}_{k, n}^{o_{i}}=\operatorname{dec}\left[\tilde{d}_{k, n}^{o_{i}}\right]$ is the decision on the corresponding symbol. Note that this decision corresponds to the input symbol at the subcarrier $k$ and time $n-\Delta_{k}$, with $\Delta_{k}$ being the equalizer's delay.
As it is well known, in a V-BLAST-type scheme, streams achieving lower mean squared detection error are extracted in earlier stages. Our equalization scheme achieves two goals simultaneously. Not only it updates the equalizer taps in an RLS manner, but also specifies, for each time instant, the ordering in which the streams must be detected. Let us assume that the equalizer of the $p$ th stage must be computed given the DFEs of the previous stages and associated symbol decisions according to the ordering $\left\{o_{1}, o_{2}, \ldots, o_{p-1}\right\}$. The remaining stream indices form the set $O_{p}(n)=\left\{1,2, \ldots, N_{t}\right\} \backslash\left\{o_{1}, o_{2}, \ldots, o_{p-1}\right\}$. To find out which of these streams achieves the lowest squared error and hence should be detected at the current stage, all the $N_{t}-p+1$ respective equalizers, $\boldsymbol{w}_{k, n}^{(r)}(p), r \in O_{p}(n)$, must be updated first. The equalizer of the $p$ th stage corresponding to the $r$ th stream, $\boldsymbol{w}_{k, n}^{(r)}(p)$, is obtained by minimizing the following least squares (LS) cost function:

$$
\mathcal{E}_{k, n}^{(r)}(p)=\sum_{l=0}^{n} \lambda^{n-l}\left|\hat{d}_{k, l}^{r}-\tilde{d}_{k, l}^{r}(p)\right|^{2}, \quad r \in O_{p}(n)
$$

where $0<\lambda \leq 1$ is the forgetting factor and, as in (1),

$$
\tilde{d}_{k, l}^{r}(p)=\Re\left\{\left[\boldsymbol{w}_{k, n}^{(r)}(p)\right]^{H} \boldsymbol{y}_{k, l}(p)\right\}=\left[\overline{\overline{\boldsymbol{w}}}_{k, n}^{(r)}(p)\right]^{T} \overline{\overline{\boldsymbol{y}}}_{k, l}(p),
$$

with

$$
\overline{\overline{\boldsymbol{y}}}_{k, l}(p)=\left[\Im\left(\overline{\boldsymbol{y}}_{k, l}\right)^{T} \Re\left(\overline{\boldsymbol{y}}_{k, l}\right)^{T} \hat{\boldsymbol{d}}_{k, l}^{T}(p)\right]^{T}
$$

and

$$
\begin{aligned}
& \overline{\overline{\boldsymbol{w}}}_{k, n}^{(r)}(p)= \\
& \quad\left[\Im\left[\boldsymbol{f}_{k, n}^{(r)}(p)\right]^{T} \Re\left[\boldsymbol{f}_{k, n}^{(r)}(p)\right]^{T} \Re\left[\boldsymbol{b}_{k, n}^{(r)}(p)\right]^{T}\right]^{T} .
\end{aligned}
$$

Note that the imaginary part of the feed-back filter is of no interest here, in accordance with the principle of [20] for OQAM equalization. The dependence of $O_{p}$ on time $n$ makes explicit the fact that the ordering may change from one time instant to the next. Regarding the $\hat{d}_{k, l}^{r}$ 's in (2), they are known symbols during the initial training period, while, after initialization, they are computed as decisions: $\hat{d}_{k, l}^{r}=\operatorname{dec}\left[\tilde{d}_{k, l}^{r}(p)\right]$. After updating all tentative equalizers at stage $p$, the one offering the lowest squared error is selected to yield the equalizer $\overline{\overline{\boldsymbol{w}}}_{k, n}(p) \equiv \overline{\overline{\boldsymbol{w}}}_{k, n}^{\left(o_{p}\right)}(p)$. That is:

$$
o_{p}=\arg \min _{r \in O_{p}(n)} \mathcal{E}_{k, n}^{(r)}(p)
$$

The computations can be significantly simplified by exploiting the DFE input structure, namely:

$$
\overline{\overline{\boldsymbol{y}}}_{k, l}(p+1)=\left[\begin{array}{cc}
\overline{\overline{\boldsymbol{y}}}_{k, l}^{T}(p) & \hat{d}_{k, l}^{o_{p}}
\end{array}\right]^{T},
$$

to arrive at order- and time-recursive ways of updating the quantities involved. This can be done in a manner analogous to that followed in [5] and hence the reader is referred to that work for details. 


\section{A. Cholesky Factorization-Based Parameterization}

A numerically stable version of the above algorithm can result by using the LS normal equations to re-parameterize the equalizers $\overline{\overline{\boldsymbol{w}}}_{k, n}^{(r)}(p)$ as

$$
\overline{\overline{\boldsymbol{w}}}_{k, n}^{(r)}(p)=\boldsymbol{R}_{k, n}^{-1}(p) \boldsymbol{v}_{k, n}^{(r)}(p),
$$

where $\boldsymbol{R}_{k, n}(p)$ is the Cholesky factor of the DFE input autocorrelation matrix,

$$
\boldsymbol{\Phi}_{k, n}(p)=\sum_{l=0}^{n} \lambda^{n-l} \overline{\overline{\boldsymbol{y}}}_{k, l}(p) \overline{\overline{\boldsymbol{y}}}_{k, l}^{T}(p),
$$

and

$$
\boldsymbol{v}_{k, n}^{(r)}(p)=\boldsymbol{R}_{k, n}^{-T}(p) \boldsymbol{z}_{k, n}^{(r)}(p),
$$

with $\boldsymbol{z}_{k, n}^{(r)}(p)$ being the corresponding cross-correlation vector, ${ }^{5}$

$$
\boldsymbol{z}_{k, n}^{(r)}(p)=\sum_{l=0}^{n} \lambda^{n-l} \overline{\overline{\boldsymbol{y}}}_{k, l}(p) \hat{d}_{k, l}^{r} .
$$

With this starting point, the algorithm can be developed in exactly the same manner as in [12] and hence the details are omitted. In addition to providing robustness to numerical inaccuracies, this scheme also enjoys computational efficiency, as discussed in [12]. Further computational savings can result, in slowly fading channels, by updating the ordering only once in a block of FBMC symbols instead of on a symbol-bysymbol basis, as suggested in [5].

The algorithm is summarized in the appendix. For the details of its development, the reader is referred to [12].

\section{Simulation Results}

The above algorithm has been tested in several scenarios. In this section, some representative simulation results are presented, for a $2 \times 2 \mathrm{FBMC/OQAM}$ system with OQPSK input. The filter banks of [3] are employed, with $M=64$ subcarriers and an overlapping factor of $K=4 .{ }^{6}$ In all cases considered, and in order to assess the gain from taking the frequency selectivity of the $M$ subchannels into account, the results of applying the DFE with the assumption of frequency flat subchannels, namely with $L_{f}=1$ and $L_{b}=0$, are also included. The equalization delay was chosen to be the same for all subcarriers and equal to $\Delta=L_{f}-1$. Frames of 106 FBMC symbols, the first 40 of which are used as (pseudorandom) training, were transmitted in the experiments. The algorithm was tested in the equalization of Rayleigh quasi-stationary ( $M$ block fading) channels of both low (Veh-A) and high (Veh-B) frequency selectivity, with both slow and fast fading.

An example of the time evolution of the mean squared symbol error (MSE) is shown in Fig. 3, for a fast fading Veh-A channel at an SNR of $25 \mathrm{~dB}$. It can be seen that, in the training

\footnotetext{
${ }^{5}$ In decision-directed mode, $\hat{d}_{k, n}^{r}$ are computed using the optimum equalizer and detection ordering found at the previous time $n-1$, i.e., $\tilde{d}_{k, n}^{o_{p}}=$ $\left[\overline{\overline{\boldsymbol{w}}}_{k, n-1}(p)\right]^{T} \overline{\overline{\boldsymbol{y}}}_{k, n}(p)$ and $\hat{d}_{k, n}^{o_{p}}=\operatorname{dec}\left[\tilde{d}_{k, n}^{o_{p}}\right]$, where $o_{p}$ here refers to the detection ordering at time $n-1$.

${ }^{6}$ Thus, the filters in the filter banks are of length $K M$
}

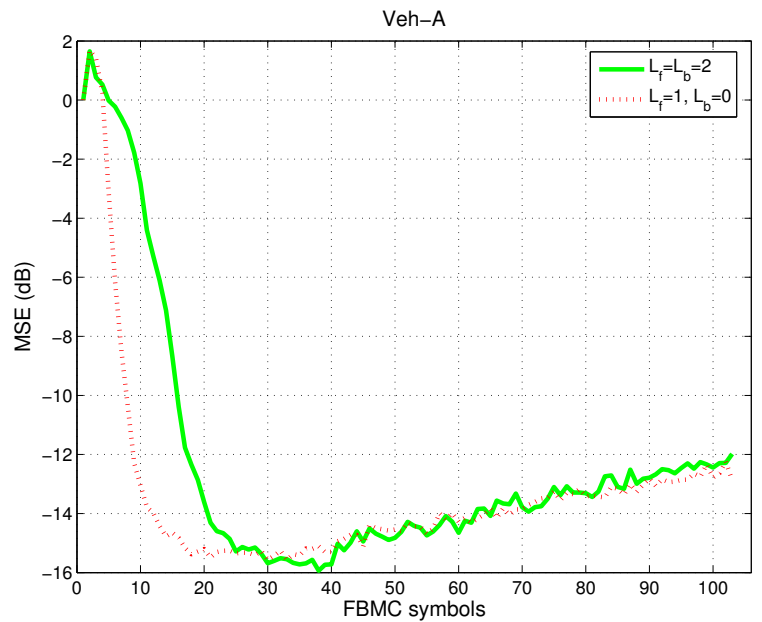

Fig. 3. Time evolution of the mean symbol squared error for a MIMOFBMC/OQAM system with $M=64, K=4,2 \times 2$ Veh-A channel, with normalized Doppler spread $1.067 \times 10^{-3}$, and equalizer parameters $L_{f}=$ $L_{b}=2$ (solid line) and $L_{f}=1, L_{b}=0$ (dotted line), and $\lambda=0.95$; $E_{b} / N_{0}=25 \mathrm{~dB}$.

period, the algorithm with short feed-forward and feed-back filters converges much faster, as expected. However, as it will be seen below, the detection performance is greatly improved when the frequency selectivity of the subchannels is taken into account, especially for highly frequency selective channels (e.g., Veh-B). Note that, after the initialization, the MSE is increasing. This is due to the effect of error propagation in the decision-directed mode and is more apparent in high mobilities.

Fig. 4 shows the (uncoded) bit error rate (BER) for VehA channels, with (a) slow and (b) fast fading. One can see that, at low and moderate SNR values, the decision errors result in higher BER for the longer equalizer and this effect is intensified at high fading rates. At higher SNRs, however, the situation changes as the frequency selectivity prevails over noise. Analogous results can be observed in Fig. 5, where VehB channels are considered. In this case of a highly frequency selective channel, the gain in BER from the use of a longer DFE is more evident and starts to hold from lower (more practical) values of the SNR.

\section{CONClusion}

The problem of adaptive channel equalization in MIMOFBMC/OQAM time-varying systems was addressed in this paper. A per subcarrier $T / 2$-spaced adaptive MIMO DFE was presented, as a result of the adaptation to the FBMC/OQAM context of an earlier algorithm originally developed for singlecarrier MIMO QAM systems. The algorithm is based on the well known equivalence of the V-BLAST idea with the generalized DFE [9] and updates both the filter taps and the BLAST ordering in an efficient RLS-based manner. It is especially suited to MIMO-FBMC/OQAM systems with highly frequency selective channels and/or a small number of subcarriers, as it can cope with the equalization of subchannels 


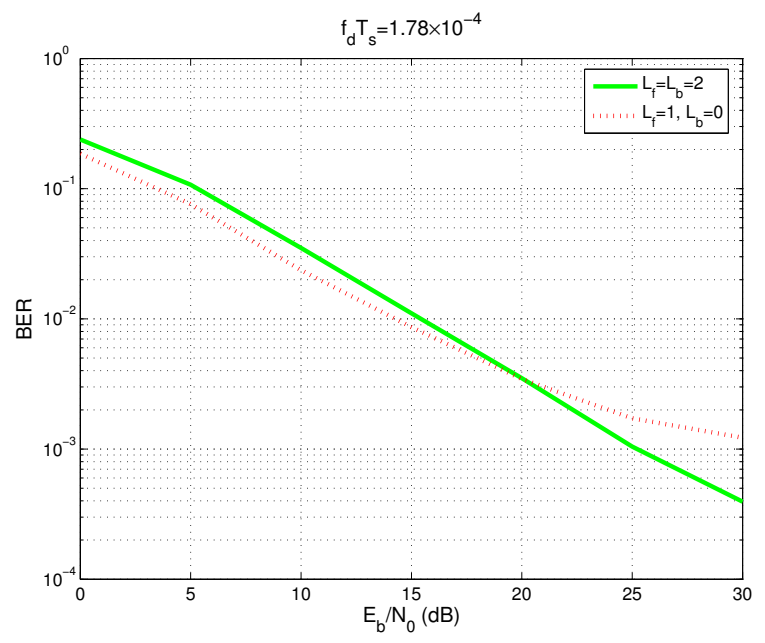

(a)

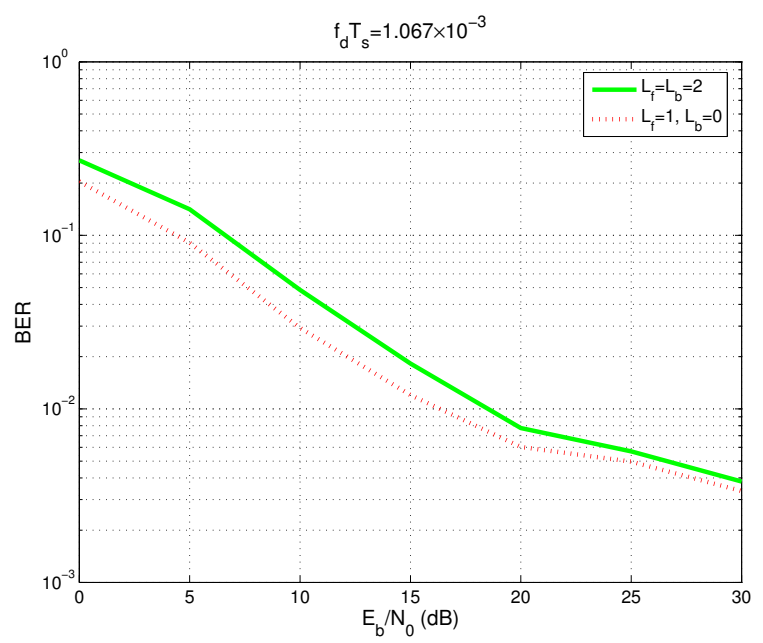

(b)

Fig. 4. BER performance for a MIMO-FBMC/OQAM system with $M=64$, $K=4,2 \times 2$ Veh-A channel, and equalizer lengths $L_{f}=L_{b}=2$ (solid line) and $L_{f}=1, L_{b}=0$ (dotted line): (a) $f_{d} T_{s}=1.78 \times 10^{-4}, \lambda=0.95$; (b) $f_{d} T_{s}=1.067 \times 10^{-3}, \lambda=0.9$.

with frequency selectivity. It inherits computational efficiency from its single-carrier counterpart and, in its square-root version, it is also numerically stable. The simulation results demonstrated its effectiveness in scenarios of varying fading rate and frequency selectivity.

For the sake of simplicity and in the vein of earlier related works [22], [10], interference from adjacent subcarriers was ignored in the development of the algorithm. The fact that there is, in practice, nonnegligible interference from neighboring subcarriers could be taken into account by augmenting the feedback structure with two more filters, one for each of the two adjacent subcarriers, as suggested (for single antenna systems) in [16]. Although this would complicate the BLAST ordering step (as explained in [10]), it is feasible provided sufficient care is taken in its implementation. It is the subject of ongoing research to develop and evaluate such an extension

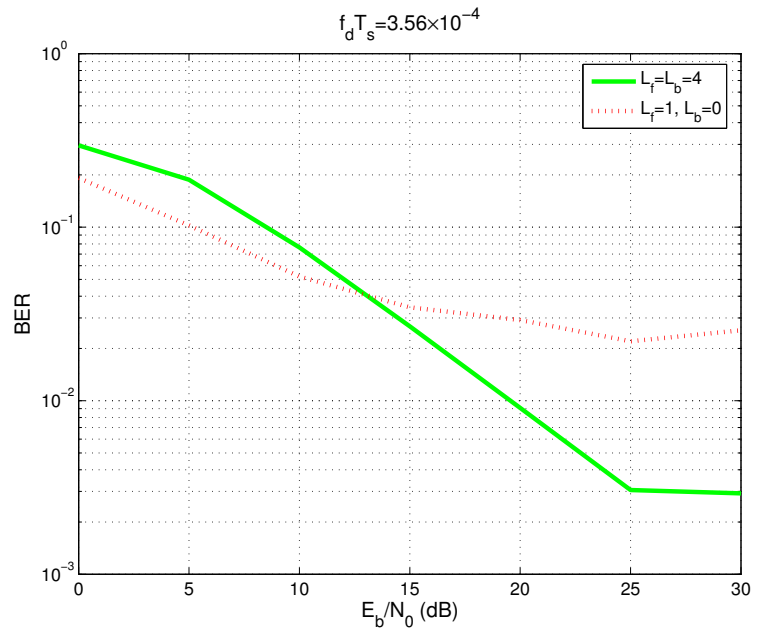

(a)

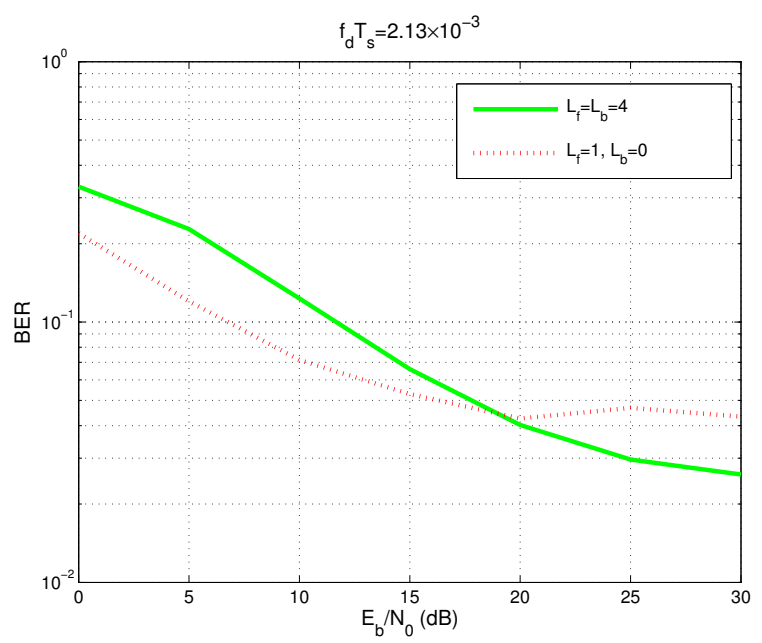

(b)

Fig. 5. As in Fig. 4, with $2 \times 2$ Veh-B channel and equalizer lengths $L_{f}=L_{b}=4$ (solid line) and $L_{f}=1, L_{b}=0$ (dotted line): (a) $f_{d} T_{s}=$ $3.56 \times 10^{-4}, \lambda=0.95$; (b) $f_{d} T_{s}=2.13 \times 10^{-3}, \lambda=0.9$.

to our algorithm. Preliminary results, though, do not show a significant improvement over the simpler version presented here.

\section{APPENDIX}

The Proposed Algorithm (For SubCARRIER $k$ )

Initialization: For $p=1, \ldots, N_{t}, o_{p}=p, \boldsymbol{v}_{k, 0}^{(p)}=\mathbf{0}$, $\mathcal{E}_{k, 0}(p)=1$. For $r=1, \ldots, N_{t}, \boldsymbol{v}_{k, 0}^{(r)}(1)=\mathbf{0} . \boldsymbol{Q}_{k, 0}=\mathbf{0}$. $\boldsymbol{R}_{k, 0}^{-1}(1)=\delta^{-1 / 2} \boldsymbol{I}$, where $\delta$ is a small positive constant.

For $n=1,2, \ldots$

(1) Compute $\boldsymbol{g}_{k, n}(1)=\boldsymbol{R}_{k, n-1}^{-T}(1) \overline{\overline{\boldsymbol{y}}}_{k, n}(1)$, and $\hat{d}_{k, n}^{o_{1}}=$ $\operatorname{dec}\left[\tilde{d}_{k, n}^{o_{1}}\right]$, where $\tilde{d}_{k, n}^{o_{1}}=\left[\overline{\boldsymbol{w}}_{k, n-1}(1)\right]^{T} \overline{\overline{\boldsymbol{y}}}_{k, n}(1)$.

(2) Find a sequence of Givens rotations $\boldsymbol{T}_{k, n}(1)$ such that

$$
\boldsymbol{T}_{k, n}(1)\left[\begin{array}{c}
-\lambda^{-1 / 2} \boldsymbol{g}_{k, n}(1) \\
1
\end{array}\right]=\left[\begin{array}{c}
\mathbf{0} \\
\alpha_{k, n}(1)
\end{array}\right]
$$


(3) Time-update the inverse Cholesky factor:

$$
\boldsymbol{T}_{k, n}(1)\left[\begin{array}{c}
\lambda^{-1 / 2} \boldsymbol{R}_{k, n-1}^{-T}(1) \\
\mathbf{0}^{T}
\end{array}\right]=\left[\begin{array}{c}
\boldsymbol{R}_{k, n}^{-T}(1) \\
\star
\end{array}\right],
$$

where $\star$ denotes "do not care" elements.

(4) For $p=2, \ldots, N_{t}$

(a) Order-update $\boldsymbol{g}_{k, n}(p)$ :

$$
\boldsymbol{g}_{k, n}(p)=\left[\begin{array}{c}
\boldsymbol{g}_{k, n}(p-1) \\
\hat{d}_{k, n}^{o p-1}-\tilde{d}_{k, n}^{o p-1} \\
\sqrt{\mathcal{E}_{k, n-1}(p-1)}
\end{array}\right]
$$

(b) Compute decisions from $\tilde{d}_{k, n}^{o_{p}}$

$$
\left[\overline{\overline{\boldsymbol{w}}}_{k, n-1}(p)\right]^{T} \overline{\overline{\boldsymbol{y}}}_{k, n}(p), \hat{d}_{k, n}^{o_{p}}=\operatorname{dec}\left[\tilde{d}_{k, n}^{o_{p}}\right] .
$$

(5) Time-update matrix $\boldsymbol{Q}_{k, n} \equiv \sum_{l=0}^{n} \lambda^{n-l} \hat{\boldsymbol{d}}_{k, l} \hat{\boldsymbol{d}}_{k, l}^{T}$ :

$$
\boldsymbol{Q}_{k, n}=\lambda \boldsymbol{Q}_{k, n-1}+\hat{\boldsymbol{d}}_{k, n} \hat{\boldsymbol{d}}_{k, n}^{T}
$$

(6) For $r=2, \ldots, N_{t}$

(a) Time-update $\boldsymbol{v}_{k, n}^{(r)}(1)$ :

$$
\boldsymbol{T}_{k, n}(1)\left[\begin{array}{c}
\lambda^{1 / 2} \boldsymbol{v}_{k, n-1}^{(r)}(1) \\
\hat{d}_{k, n}^{r}
\end{array}\right]=\left[\begin{array}{c}
\boldsymbol{v}_{k, n}^{(r)}(1) \\
\star
\end{array}\right]
$$

(b) $\mathcal{E}_{k, n}^{(r)}(1)=\left[\boldsymbol{Q}_{k, n}\right]_{r, r}-\left\|\boldsymbol{v}_{k, n}^{(r)}(1)\right\|^{2}$

(7) Set $\mathcal{E}_{k, n}(1)=\min _{r} \mathcal{E}_{k, n}^{(r)}(1)$ and let $\boldsymbol{v}_{k, n}(1)$ be the corresponding $\boldsymbol{v}_{k, n}^{(r)}(1)$.

(8) For $p=2, \ldots, N_{t}$

(a) For $r \in O_{p}(n)$

(i) Order-update $\boldsymbol{v}_{k, n}^{(r)}(p)$ :

$$
\boldsymbol{v}_{k, n}^{(r)}(p)=\left[\begin{array}{c}
\boldsymbol{v}_{k, n}^{(r)}(p-1) \\
\frac{\left[\boldsymbol{Q}_{k, n}\right]_{o_{p-1}, r}-\boldsymbol{v}_{k, n}^{T}(p-1) \boldsymbol{v}_{k, n}^{(r)}(p-1)}{\sqrt{\mathcal{E}_{k, n}(p-1)}}
\end{array}\right]
$$

(ii) $\mathcal{E}_{k, n}^{(r)}(p)=\mathcal{E}_{k, n}^{(r)}(p-1)-\left|\left[\boldsymbol{v}_{k, n}^{(r)}(p)\right]_{\text {end }}\right|^{2}$, where $\left[\boldsymbol{v}_{k, n}^{(r)}(p)\right]_{\text {end }}$ is the last element of $\boldsymbol{v}_{k, n}^{(r)}(p)$.

(b) Set $\mathcal{E}_{k, n}(p)=\min _{r} \mathcal{E}_{k, n}^{(r)}(p)$ and let $\boldsymbol{v}_{k, n}(p)$ be the corresponding $\boldsymbol{v}_{k, n}^{(r)}(p)$.

\section{REFERENCES}

[1] P. Amini, R. Kempter, and B. Farhang-Boroujeny, "A comparison of alternative filterbank multicarrier methods for cognitive radio systems," Proc. SDR-2006, 13-17 Nov. 2006, Orlando, FL.

[2] J. G. Andrews, A. Ghosh, and R. Muhamed, Fundamentals of WiMAX: Understanding Broadband Wireless Networking, Prentice-Hall, 2007.

[3] M. G. Bellanger, "Specification and design of a prototype filter for filter bank based multicarrier transmission," Proc. ICASSP'01, vol. 4, pp. 2417-2420, 7-11 May 2001, Salt Lake City, UT.

[4] N. Benvenuto, G. Cariolaro, G. Michieletto, and L. Vangelista, "Analysis of channel noise in orthogonally multiplexed QAM systems," Proc. GLOBECOM'93, vol. 3, pp. 1990-1995, 28 Nov.-2 Dec. 1993, Houston, TX.

[5] J. Choi, H. Yu, and Y. H. Lee, "Adaptive MIMO decision feedback equalization for receivers with time-varying channels," IEEE Trans. Signal Processing, vol. 53, no. 11, pp. 4295-4303, Nov. 2005.
[6] M. El Tabach, J. P. Javaudin, and M. Hélard, "Spatial data multiplexing over OFDM/OQAM modulations," Proc. ICC'07, pp. 4201-4206, 2428 June 2007, Glasgow, UK.

[7] G. J. Foschini, G. D. Golden, R. A. Valenzuela, and P. W. Wolniansky, "Simplified processing for high spectral efficiency wireless communication employing multi-element arrays," IEEE Journal on Selected Areas in Communications, vol. 17, no. 11, pp. 1841-1852, Nov. 1999.

[8] G. Garbo, S. Mangione, and V. Maniscalco, "Wireless OFDM-OQAM with a small number of subcarriers," Proc. WCNC'08, pp. 187-192, 31 March-3 April 2008, Las Vegas, NV.

[9] G. Ginis and J. M. Cioffi, "On the relation between V-BLAST and the GDFE," IEEE Communications Letters, vol. 5, no. 9, pp. 364-366, Sept. 2001.

[10] A. Ikhlef and J. Louveaux, "Per subchannel equalization for MIMO FBMC/OQAM systems," Proc. PACRIM'09, pp. 559-564, 2326 Aug. 2009, Victoria, BC.

[11] J.-P. Javaudin, D. Lacroix, and A. Rouxel, "Pilot-aided channel estimation for OFDM/OQAM,' Proc. VTC'03 (Spring), 26-29 April 2003, Jeju Island, Korea.

[12] V. Kekatos, A. A. Rontogiannis, and K. Berberidis, "Cholesky factorization-based adaptive BLAST DFE for wideband MIMO channels," EURASIP Journal on Advances in Signal Processing, vol. 2007, Article ID 45789.

[13] B. Le Floch, M. Alard, and C. Berrou, "Coded orthogonal frequency division multiplex," Proc. IEEE, vol. 83, no. 6, pp. 982-996, June 1995.

[14] H. Lin and P. Siohan, "A new transceiver system for the OFDM/OQAM modulation with cyclic prefix," Proc. PIMRC'08, 15-18 Sept. 2008, pp. 1-5, Cannes, France.

[15] B. Mongol et al., "MIMO zero-forcing equalizer for BFDM/OQAM systems in the highly mobile environments," Proc. GLOBECOM'06, pp. 1-5, 27 Nov.-1 Dec. 2006, San Fransisco, CA.

[16] S. Nedic and N. Popovic, "Per-bin DFE for advanced OQAM-based multicarrier wireless data transmission systems," Proc. 2002 Int'l Zürich Seminar on Broadband Communications, pp. 38-1-38-6, 1921 Feb. 2002, ETH Zürich, Switzerland.

[17] A. A. Rontogiannis, V. Kekatos, and K. Berberidis, "A square-root adaptive V-BLAST algorithm for fast time-varying MIMO channels," IEEE Signal Processing Letters, vol. 13, no. 5, pp. 265-268, May 2006.

[18] P. Siohan, C. Siclet, and N. Lacaille, "Analysis and design of OFDM/OQAM systems based on filterbank theory," IEEE Trans. Signal Processing, vol. 50, no. 5, pp. 1170-1183, May 2002.

[19] G. L. Stüber et al., "Broadband MIMO-OFDM wireless communications," Proc. IEEE, vol. 92, no. 2, pp. 271-294, Feb. 2004.

[20] J. C. Tu, "Optimum MMSE equalization for staggered modulation," Proc. 27th Asilomar Conf. Signals, Systems, and Computers, pp. 14011406, 1-3 Nov. 1993, Pacific Grove, CA.

[21] R. van Nee and R. Prasad, OFDM for Wireless Multimedia Communications, Artech House Publ., 2000.

[22] D. S. Waldhauser, L. G. Baltar, and J. A. Nossek, "Adaptive decision feedback equalization for filter bank based multicarrier systems," Proc. ISCAS'09, pp. 2794-2797, 24-27 May 2009, Taipei, Taiwan. 